\title{
Using GPS and Google Maps for Mapping Digital Postal Address (GhanaPostGPS)
}

\author{
Silas Kwabla Gah \\ University of Ghana \\ Department of Computer Science \\ Legon. Ghana
}

\author{
Ferdinard Katsriku, PhD \\ University of Ghana \\ Department of Computer Science \\ Legon, Ghana
}

\author{
Nana Kwame Gyamfi \\ Kumasi Technical University \\ Kumasi, Ghana \\ Department of Computer Science
}

\begin{abstract}
Google map is a platform that gives visual representation of geographical locations on the planet earth. Google maps has many features that for displaying maps and adding external contents to the map. In recent years many institutions and organizations have customized the features and functions of Google maps to build new applications that address their specific needs. Developing nations are faced with booming population growth, inadequate infrastructure and services. To provide many important services, especially financial services it is required that people are accurately located by use of a verifiable address. Developing an effective addressing system has been a challenge for many developing nations due to inadequate road and street network. This paper discusses the use of Google maps API to link properties in Ghana, hence assigning a digital address to each landmark in the country. The paper examines the technology that Google maps API provides and how it was harnessed to develop the GhanaPostGPS addressing system. This application helps users to acquire their digital address and enables others to search the location of an address via the system. The result from the Google maps API reverse geocoding shows that there is no district in the JSON response, an indicating factor showing extra work done by the developers of the application. This clearly shows the work is not a direct replica of services offered by Google maps although some services of Google maps were employed.
\end{abstract}

\section{Keywords}

Google Maps API, Google Maps, Digital address, Global Positioning System (GPS).

\section{INTRODUCTION}

Like many developing nations Ghana has serious problems with infrastructure. Due to inadequate road and street network many properties lack addresses. An accurate and verifiable addressing system is important in the provision of many services. The Government of Ghana in its quest overcome some of the challenges in the provision of services looked to use of digital technology and to develop a system that will help in the allocation of a digital address to every single $5 \mathrm{~m}$ by $5 \mathrm{~m}$ landmark in the country. There are a wide range of Global Position System (GPS) that may be employed to easily help in the allocation of a precise and accurate position to every specific $5 \mathrm{~m}$ by $5 \mathrm{~m}$ grid. An example of such a global system is what 3 words [1]. In order to provide digital address system for Ghana, coordinates from satellite position systems can be used to help give an accurate position of an. appropriately chosen square grid anywhere within the country. According to [1], coordinates system which is used to showcase the actual location of a landmark on a mappping system are of various type. Google employs the Word
Geodetic System 84 (WGS 84) which is the exact one Global Position System employs as well. The coordinates are expressed as latitude and longitude value. While the latitude determines the measure from south to north the longtitude measures from west to east. The point of zero value for the latitude coordinates is called the equator. All coordinates below the equator normally have a negative value called the southern hemisphere and similarly the northern hemisphere has positive coordinate values. Again there is a point of zero coordinate for the longitude and is called the prime meridian. This results in all positions or coordinates to the east of this line to have a positive value and to the west of this line to have a negative value. The coordinate values are usually expressed as two comma separated values in decimals format, with the latitdue value preceding the longitude value.

There are however a number of mapping system in use currently. A research conducted by [2], indicated that there is not much difference in the accuracy of the three mapping system that were compared, these are the Google maps, OpenStreetMap and Bing Maps.

\section{GOOGLE MAPS API}

An API provides an interface that allows developers to tap into a framework developed by a third party. According to documentation at [3] google maps API provides software developers with an efficient way of embedding earth maps into web pages and also provides the ability to retrieve data from the google maps framework. The API is supported on the web and on mobile platforms as well. Among the services the web API endpoint provides are map directions, distance matrix between two points, map elevation, geocoding, geolocation, roads, and time zone and google places. For the mobile API, services include google maps android, google places for android, google maps software development kit for iOS, google places API for iOS. Google Maps API introduced a version 3 of its API which enables developers to create a map. A sample of the result is shown in figure 1, the display shows view of the current location of the user in Accra, Ghana.

\subsection{Embed Map}

In order to embed google maps into a web application or mobile application, all that one needs to do is to add a reference to the google API key which is used by google to identify the application. A good example of this is shown in figure 1. The Fig 2 shows the code for embedding google maps API into a web page and Fig 3 and Fig 4 shows the scenario for embedding google maps API into an android and iOS applications respectively.

In Figure 2, the code seeks to display the location with this coordinates (lat: -25.16 , lng: 131.044). This is added to a 
simple html page with nothing but a style and a div to hold the map to be displayed.

The snippet code of Figure 3, shows how to display a google map on an android device with the coordinates of (lat:-33.852, lng: 151.211). The activity class implements a method called "OnMapReadyCallback", this is to help the asynchronous nature of calling the map and help run the code in the background that way preventing the hanging of the main thread of the application. A title is given to the mark displayed on the map.

In Figure 4, shows the sample code for the iOS version in displaying the google map on the platform.

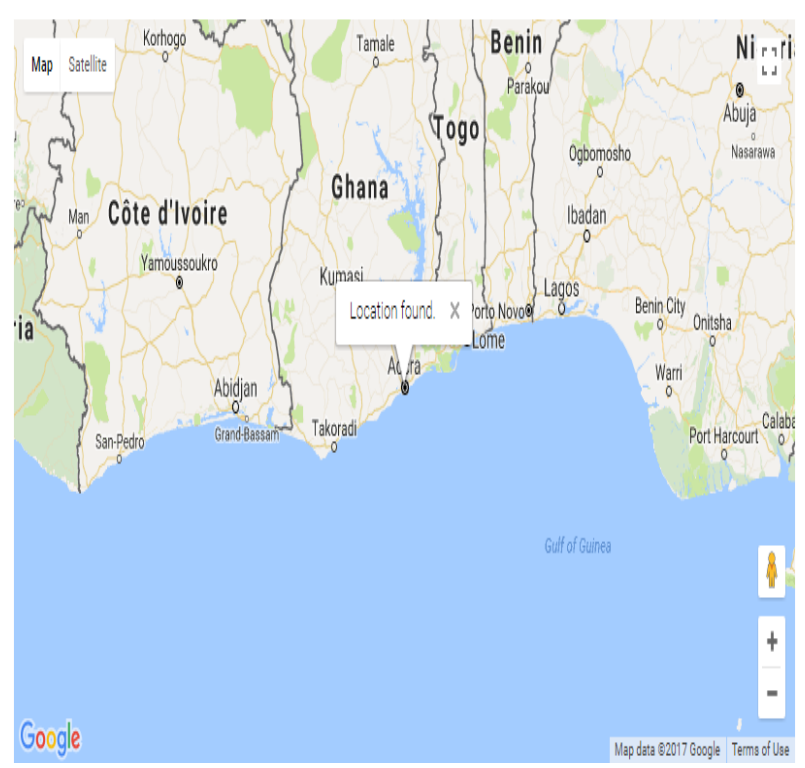

Fig 1 Google Maps User Current Location

\section{DIGITAL ADDRESS DESIGN}

The GhanaPostGPS system according to developers is a digital addressing system that seeks to provide each landmark or property with a unique digital address. The idea is to divide the country into $5 \mathrm{~m}$ by $5 \mathrm{~m}$ square grid with each grid being assigned a unique digital address. With this platform every single landmark or property can be located on a mapping system such as google map. Google Maps has become generally very popular because it has been made very easy to integrate and even build customizable map content and imagery using the version 3 of Google Maps. According to [4] the researchers were able to develop a web-based application based on Google Maps which enable visitors and student of the UA campus to easily and freely navigate the campus using this application. The application relied on a MySQL database server which stored all the locations on campus and employed the services of Google Maps APIs which enable the easy presentation on map.

On first launching of the web version of the GhanaPostGPS application, a default location of 19 Patrice Lumumba RD, Accra. Ghana with the digital address of GA-059-3733. Users of the application can search for a new location via a search space provided.

In [3] the identification of the geographic location of a user device using a number of techniques is described. Generally geolocation services employs the servives of networking routing addresses or the internal global positioning system
(GPS) to pin point the user location. This feature of geolcation is device-specific API, which informs us that the device or browers must support geolocation in order to make accessing the user current location a possibility. The W3C provides geolocation API specification in order to obtain the user current or device location. According to W3C the API does not actually know the underlying location information sources, as this could come from a number of sources such as IP address, RFID, WiFi. Figure 5 shows the number of browsers that support provision of user current locations.

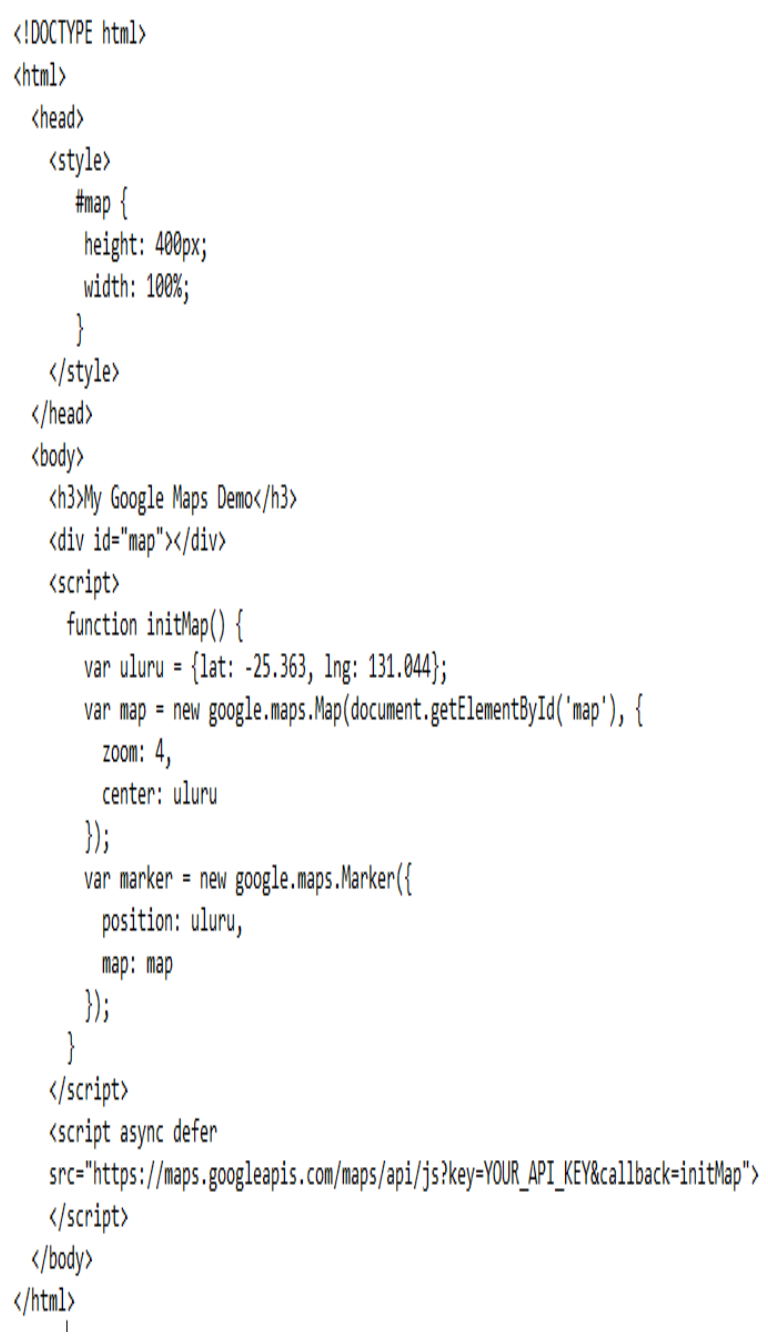

Fig 2 Code for embedding google maps into web application 
package con, example, nopithitharken;

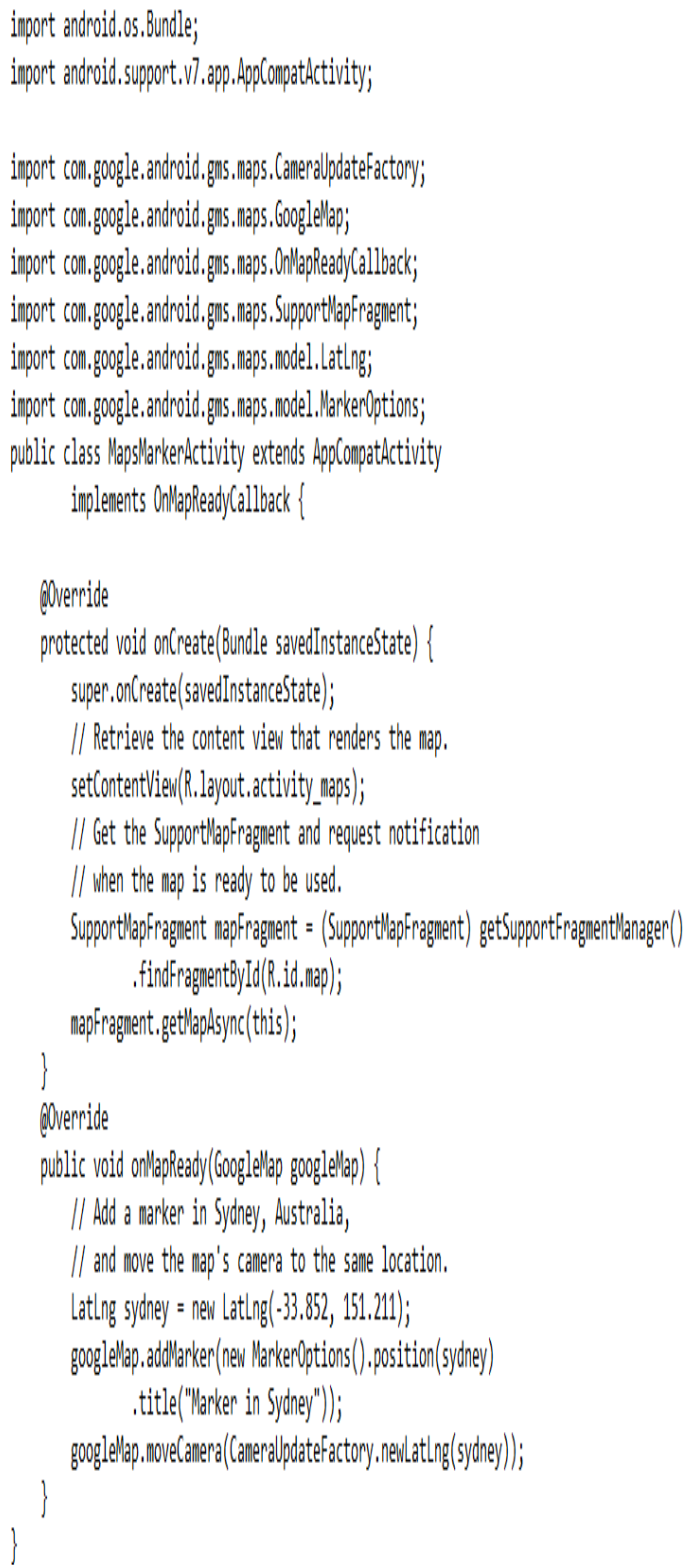

Fig 3 Code for embedding google maps into an android application

\begin{tabular}{|c|c|c|c|c|c|}
\hline API & $\theta$ & e & 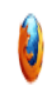 & 0 & 0 \\
\hline Geolocation & $\begin{array}{c}5.0 \cdot 49.0 \text { (htotp) } \\
50.0 \text { (htops) }\end{array}$ & 9.0 & 3.5 & 5.0 & 16.0 \\
\hline
\end{tabular}

Fig 4 Browser support geolocation

\subsection{Ghanapostgps Components}

To accomplish the task of displaying all regions and districts across the country, one can think of adding these locations onto the Google Maps. Interestingly Google has a number of ways for developers to incorporate their own data onto the map. These information can be stored on a secured backend and retrieved via an API.

The GhanaPostGPS gives users of the application the room to get their current location and this is saved in a secured backend as the landmark location of the user of the application. The Google maps API gives developers the ability to add dynamic maps to their content either mobile or web pages and even overlay these points. Google Maps API provides a Data layer container for adding geospatial data which one can customize or even display GeoJSON data on the map. According to google GeoJSON is a standard for geospatial data provided on the internet. GeoJSON are normally light weight and makes it much easier in handling such data on the internet. The data layer of the Google API makes it possible to load and display these points on a map. The Figure 5 shows a sample from google documentation on how GeoJSON information can be used.

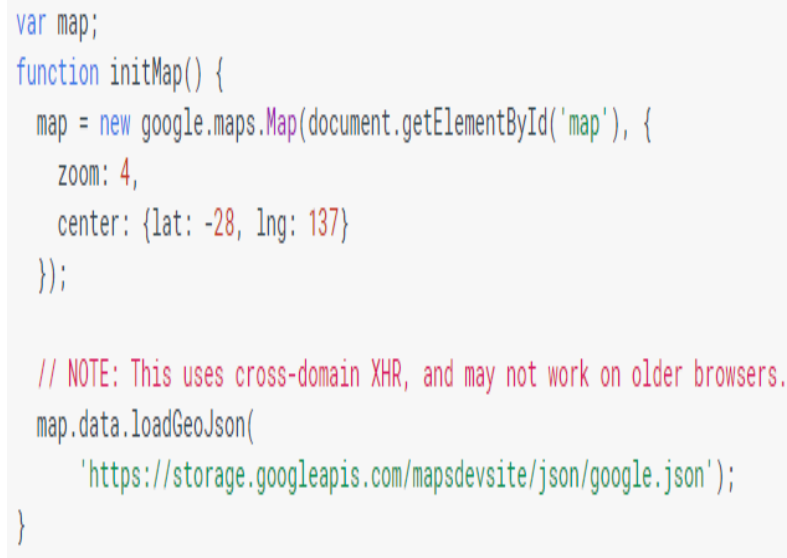

I/ NOTE: This uses cross-domain XhR, and may not work on older browsers. map. data, loadGeoJson(

Fig 5 GeoJSON Data layer sample

The Google map API also allows display of XML geospatial data as well on the google maps. Such data as XML can be overlaid within maps from an external source or KML files. These XML can also be retrieved from a backend, even though there are no standards on these XML schema.

According to research by [5], ability to display geodata information stored in a secured backend can easily be achieved by employing XML or KML overlays. This way any predefined location such as hotels, businesses can be configured and displayed on any mapping system of choice.

Figure 6, shows the web view of the GhanaPostGPS which when launched for the first time gets the user current location and displays it on the map. Since every client with such a browser or mobile device has an embedded GPS locator which is used to get the current location (longitude and latidue coordinates). This way when the user clicks on get Digital Address button on the form, the Region and District from which the user is located is concantenated and hyphenated along with some unique figures to allocated the user a diigital address.

The various Region and District are all known ahead and captured, sample of these values are shown in the Figure 7. This way google services of reverse geocoding API helps in getting the Region name and District names as soon as the API is called with the specified coordinates. Geocoding is the process of converting an address into its geographical coordinates that is the longitude and latitude, and reverse 
geocoding is the way of converting a geographical coordinates into a human readable address in a JSON format.

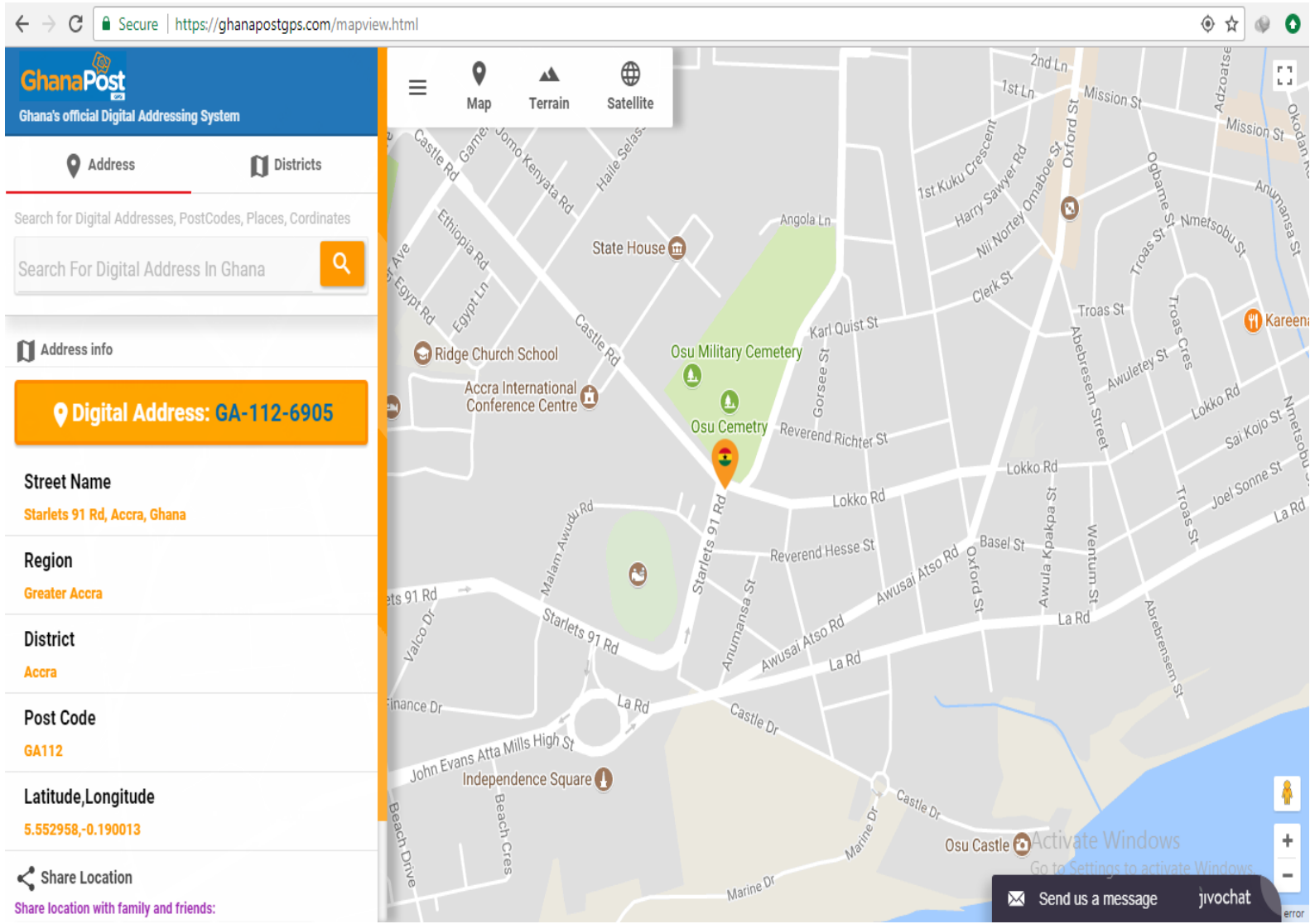

Fig 6 GhanaPOSTGPS Web View

\section{Ashanti Region}

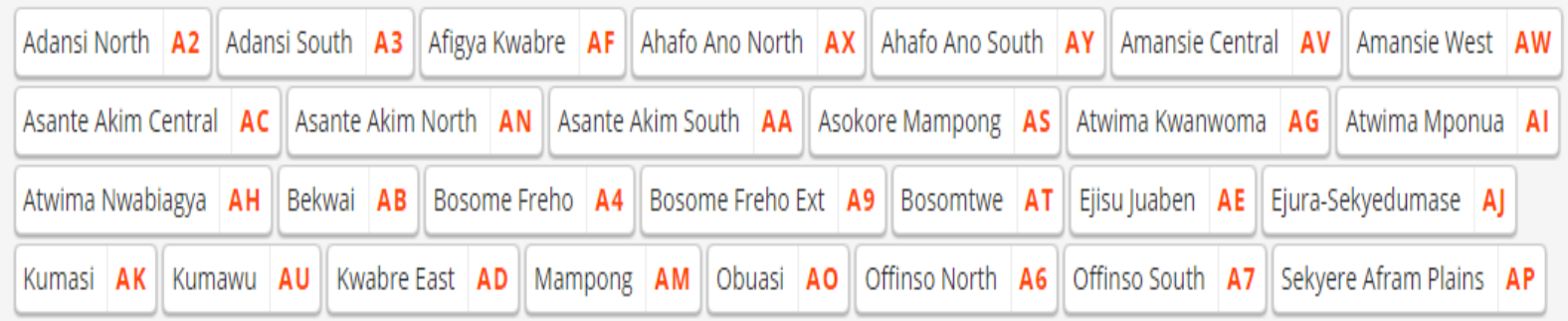

Sekyere Central AQ Sekyere East AR Sekyere South AZ

\section{Fig 7 Ashanti Region District Break Down}

This way when the user clicks get Digital Address, the user's current geolocation is used to acquire the address name which contains the users Region and District code. Figure 8 shows the iOS view of the GhanaPostGPS and the string of digits shown in green is the generated digital address based on the user's current location. 


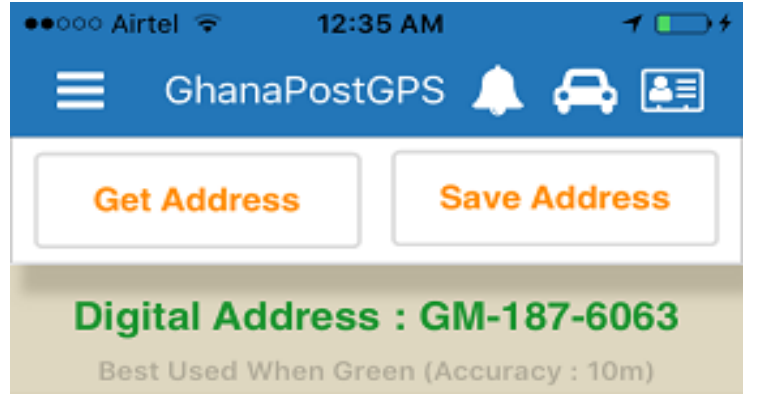

\section{Street Name}

Oyarifa Rd, Ghana

\section{Region Name \\ Greater Accra}

\section{District Name}

La Nkwantanang-Madina

\section{Postal Code}

GM187

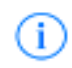

\section{Latitude Longitude}

$5.76245,-0.18142$

9 Map (2) Route 藏 Street \& Share

Fig 8 Reverse Geocoding

Figure 8 also shows the iOS interface of the GhanaPostGPS, the latitude and longitude values when applied to the Google maps API reverse geocoding shows the values given in Figure 10 in JSON format as response.

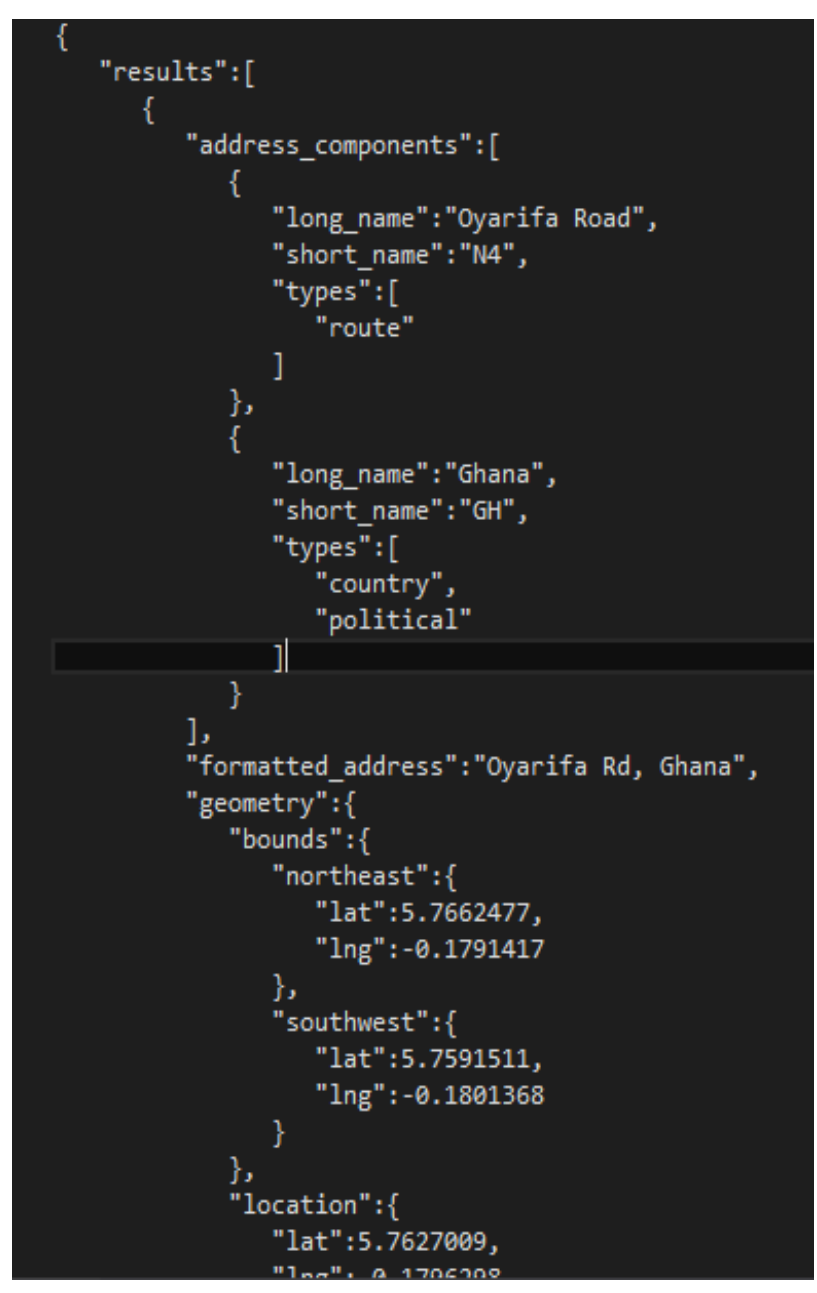

Fig 9 Reverse Geocoding component one 


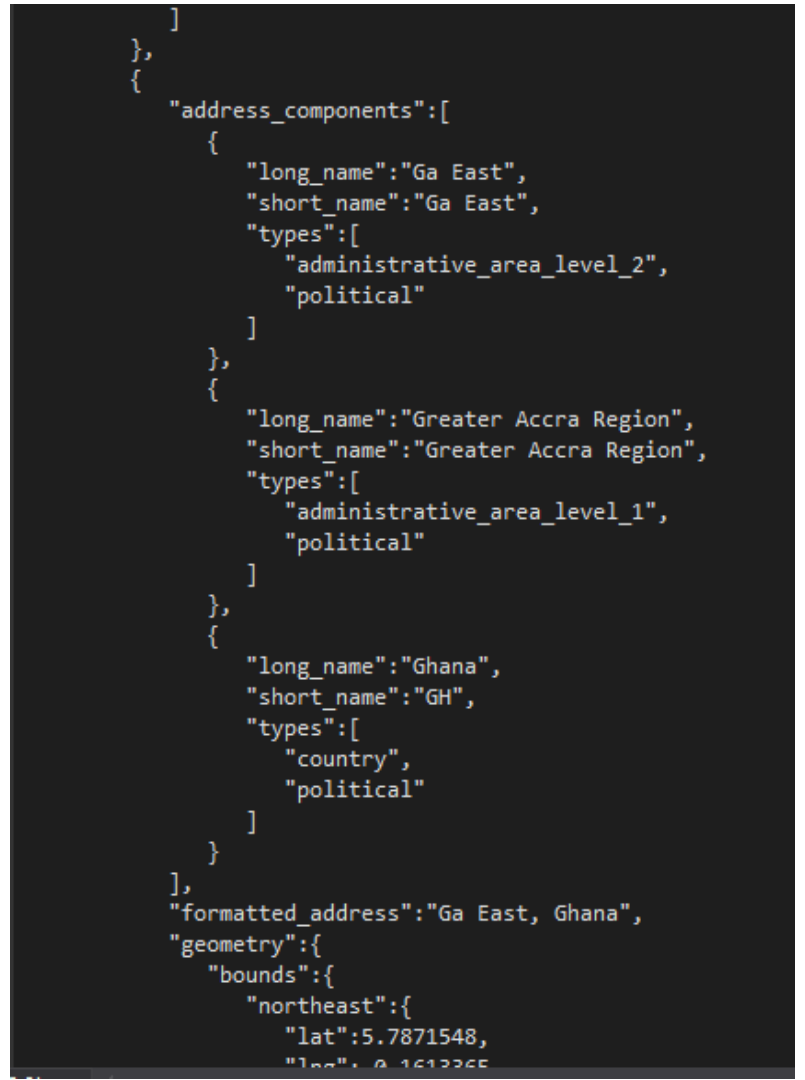

Fig 10 Reverse Geocoding component two

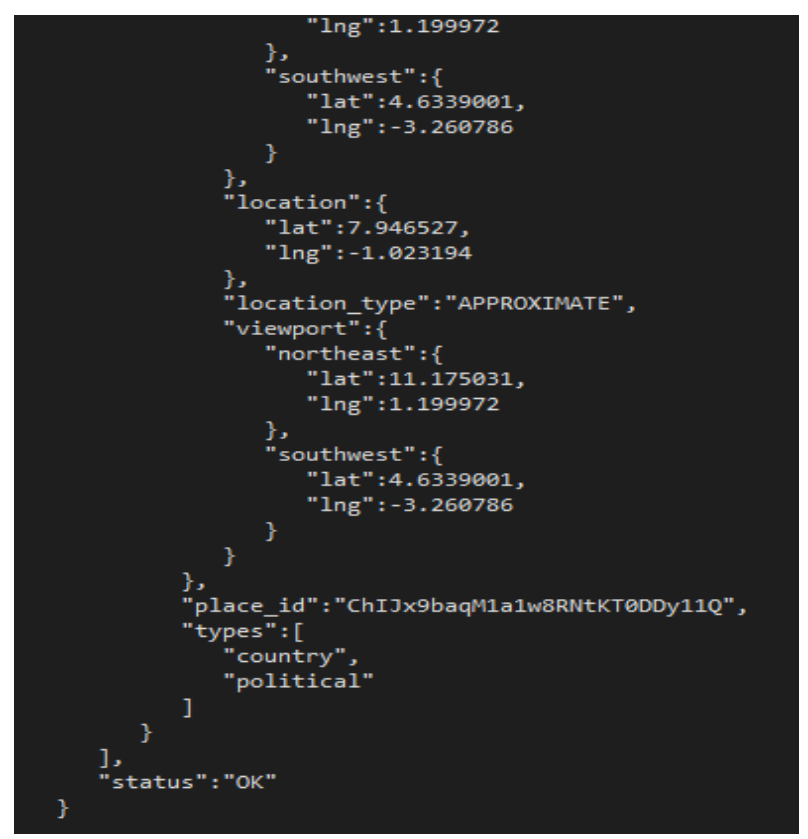

Fig 11 Reverse Geocoding response status
In order to get this response one needs to supply a valid latitude and longitude coordinates and google application key. The key is mainly for quota management and billing when request to the service elapses the stated time. The response status in Figure 11 shows that the response returned was a success and other status of failure is documented on google page.

The service returns an array of items placed in the result as shown in Figure 9 and 10. The result array normally contains zero or more results. The result array contains the fields' types [].

\section{CONCLUSION}

The result from the research shows clearly that there is clear case of work done to add up to what Google maps API provides. The result from the reverse geocoding shows no address component of district but at least the region is shown in the array of result. This shows that GhanaPostGPS has taken the pain in adding all the region and district in the country and overlay this result on Google maps, this way taking advantage of the Google maps platform. Notwithstanding this work done, it is the view that the amount that went into paying for this package is outrageous. More so the mobile application is of type hybrid and not a native application. This does not make it as quality mobile application as that of a native one.

\section{REFERENCES}

[1] Gabriel Svennerberg, Beginning Google Maps API 3, USA: Apress, 2010.

[2] R. J. A. W. P. M. Blazej Cipeluch, "Comparison of the accuracy of OpenStrretMap for ireland with Google Maps and Bing Maps," in Proceedings of the Ninth International Symposium on Spatial Accuracy Assessment in Natural Resuorces and Enviromental Sciences, Ireland, 2010.

[3] Google, "Google Maps API," Google, 10 December 2017. [Online]. Available: https://googledevelopers.appspot.com/maps/faq\#whatis. [Accessed 10 2017 2017].

[4] H. Z. J. K. E. C. Hoang Nguyen, "RooWay: A Webbased Application for UA Campus," in International Conference on Computational Science and Computational Intelligence, 2015.

[5] I. O. \&. U. N. N. Bildirici, "Web mapping with Google maps mashups: overlaying geodata," in A special joint symposium of ISPRS technical commission IV \& AutoCarto in conjunction with ASPRS/CaGIS 2010 fall specialty conference, Orlando, FL (pp. 15-19)., Orlando, 2010. 